Received: 19 December 2016

Accepted: 22 May 2017

Published online: 03 July 2017
ENTIFIC REP

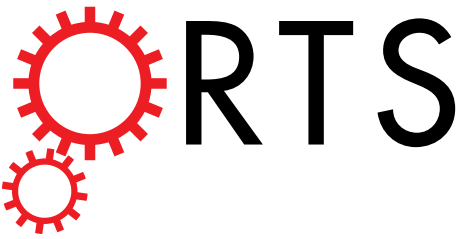

OPEN

\section{Comparison of laboratory testing methods for the diagnosis of tuberculous pleurisy in China}

\author{
Qing Zhang ${ }^{1,2} \&$ Caicun Zhou ${ }^{1,3}$
}

To evaluate the diagnostic utilities of different methods for Mycobacterium tuberculosis (M. tuberculosis) detection in pleural fluid that represent potentially cost-effective measures for resource-limited settings in China. We compared diagnostic characteristics of the AmpSure simultaneous amplification and testing method, the BACTEC MGIT 960 system, and acid-fast bacilli staining of specimen smears for detection of $M$. tuberculosis in pleural fluids of 438 patients with suspected tuberculous pleurisy. Chest radiographs, computed tomography scans and the results of sputum and pleural biopsy testing were used for confirmations of tuberculosis diagnoses. The sensitivity of the AmpSure test (21.2\%) was significantly higher than that of smear analysis (3.1\%; $p<0.001)$, but was similar to that of the BACTEC culture method (17.8\%; $p>0.05)$. The specificity of the AmpSure, BACTEC, and smear tests were $100 \%$, $96.4 \%$, and $100 \%$, respectively. The positive and negative predictive values of the AmpSure, BACTEC, and smear tests were $100 \% / 23.1 \%$; $78.6 \% / 19.8 \%$; and $100 \% / 22.4 \%$, respectively. The sensitivity of ADA, IFN $\gamma$ and histopathological analyses of pleural biopsies were all $100 \%$. The sensitivities of all three methods were suboptimal for the detection of $M$. tuberculosis in pleural fluid. Future studies of a composite diagnostic index consisting of a combination of these tests are warranted.

Although the incidence of tuberculosis (TB) has decreased during the previous decade, it remains a major cause of morbidity and mortality worldwide, with the greatest disease burden occurring in Asia and Africa ${ }^{1}$. Tuberculous pleurisy (TP) is a common presentation of extrapulmonary Mycobacterium tuberculosis infection, which occurs secondary to pulmonary $\mathrm{TB}^{2}$. In TP patients, the rupture of subpleural caseous foci causes tuberculous pleural effusion (TPE), which most often occurs 6-12 weeks following primary $M$. tuberculosis infection, resulting in variable numbers of tubercle bacilli invading the pleural space ${ }^{3,4}$. Currently available laboratory tests for M. tuberculosis detection are suboptimal for diagnosing pleural extrapulmonary $\mathrm{TB}^{5}$. The ability to rapidly detect $M$. tuberculosis in pleural fluid (PF) could provide a valuable diagnostic tool for identifying TP cases.

Bacteriological detection of M. tuberculosis in pleural biopsies can fail to identify up to $45 \%$ of patients with $\mathrm{TPE}^{4}$, and methods for culturing $M$. tuberculosis take 2-5 weeks to complete ${ }^{4,6,7}$, which can delay effective medical interventions. Approximately $33 \%$ of TP patients have a negative tuberculin skin test ${ }^{4}$, and as many as $75 \%-$ $80 \%$ of patients with TPE test negative for acid-fast bacilli (AFB) in PF smear tests ${ }^{4,8}$. The detection of elevated biomarkers of $M$. tuberculosis infection in PF samples ${ }^{9-11}$ and the presence of parenchymal lesions associated with $\mathrm{TP}^{12}$ have also been investigated for TP diagnosis, but these methods require bacteriological confirmation. The Centers for Disease Control and Prevention (USA) first recommended nucleic acid amplification for TB diagnosis in $2000^{13}$, and the identification of M. tuberculosis based on RNA detection and quantification has gained favor in recent years due to lower false-positive rates ${ }^{14}$.

The AmpSure diagnostic test for M. tuberculosis (Shanghai Rendu Biotechnology, Shanghai China) is based on a previously described simultaneous amplification and testing method for $M$. tuberculosis detection ${ }^{15}$ that uses isothermal RNA amplification and real-time fluorescence detection. The AmpSure assay has demonstrated high levels of sensitivity and specificity for the diagnosis of pulmonary TB based on the analysis of sputum and bronchial lavage fluid ${ }^{16,17}$. However, the efficacy of the AmpSure assay for detecting tubercle bacilli in PF has not been reported. Our current study aimed to evaluate the diagnostic accuracy of the AmpSure assay for identifying patients with TPE, and to optimize the sensitivity of the assay for detecting M. tuberculosis in PF.

${ }^{1}$ Soochow University, Suzhou, 215006, China. ${ }^{2}$ Tuberculosis Clinic and Research Center, Shanghai Key Lab of Tuberculosis, Shanghai Pulmonary Hospital, Tongji University School of Medicine, Shanghai, 200433, China. ${ }^{3}$ Department of oncology, Shanghai Pulmonary Hospital, Tongji University School of Medicine, Shanghai, 200433, China. Correspondence and requests for materials should be addressed to C.Z. (email: Zhoucaicun121316@163.com) 


\section{Methods}

Patients. Our study was approved by the Ethics Committee of Shanghai Pulmonary Hospital, and was performed in accordance with the Declaration of Helsinki with regard to ethical principles for research involving human subjects. Written, informed consent was obtained from all of the patients prior to participation in our study.

We prospectively screened patients who had been admitted to our hospital for suspected TP between January 2014 and April 2015. Data regarding age; sex; history of TB contacts; history of TB symptoms; current TB symptoms; history of medical treatment for TB; comorbidities; and concurrent medical therapies were obtained from each patient using a standardized questionnaire. The exclusion criteria for enrollment were as follows: $<17$ years of age; seropositive for HIV; differential diagnosis of transudative pleural effusion; and a history of previous medical treatment for TB. Enrolled patients for whom a clear diagnosis could not be made after examination and treatment were excluded from our analysis.

Patients were assigned to the TB or non-TB group based on a diagnosis of TB, which required chest radiography or computed tomography $(\mathrm{CT})$ features consistent with fluid patterns in exudative pleural effusion ${ }^{18}$ and at least one of the following criteria: $M$. tuberculosis detected in PF or sputum; histological findings of caseating granulomas based on examination of pleural biopsy specimens; and the absence of other diseases that could have caused $M$. tuberculosis invasion of the PF. Patients for whom these diagnostic criteria were not satisfied were assigned to the non-TB group.

Examinations. Each patient underwent physical examination, chest radiography or CT, and guided thoracentesis using ultrasound or CT. Pleural biopsy was performed as needed. For all PF samples, an adenosine deaminase (ADA) analysis and the blood T-SPOT.TB interferon-gamma release assay were performed, and $M$. tuberculosis detection was performed using the AmpSure assay, AFB smear, and bacteriological analysis. Sputum specimens were subjected to AFB smear and bacteriological analysis only. The diagnostic testing and histopathological analysis were performed at the TB reference laboratory in Shanghai Pulmonary Hospital using routine quality control procedures. Bacteriological analysis was performed using the BACTEC MGIT 960 system (BD Life Sciences, Franklin Lakes, NJ, USA), according to the guidelines of the World Health Organization ${ }^{19}$. The AmpSure assay was performed according to the manufacturer's protocol, as previously described ${ }^{16}$. The ADA was analyzed using a colorimetric assay (Diazyme Laboratories, Poway, CA, USA) with a cut-off value of $40 \mathrm{IU} / \mathrm{mL}$, and the blood T-SPOT.TB interferon-gamma (IFN $\gamma$ ) release assay was performed as previously described ${ }^{20}$, with a cut-off value for IFN $\gamma$-positive lymphocytes of $50 \%$. Pleural biopsy specimens were examined by a qualified pathologist.

Statistical analyses. The statistical analysis was conducted using the SPSS, version 18.0 software (IBM, Armonk, NY, USA). Continuous variables are reported as the mean \pm standard deviation and range, and categorical variables are reported as the number and percentage of observations. Differences in the continuous and categorical variables were evaluated using a two-tailed Fisher exact test or a Pearson chi-squared analysis where appropriate.

The sensitivity, specificity, positive predictive value (PPV), negative predictive value (NPV), and diagnostic accuracy of the AmpSure assay for detecting M. tuberculosis in PF were calculated. Sensitivity was defined as the number of patients with a positive test result divided the number of patients in the TB group. Specificity was defined as the percentage of non-TB patients with a negative result. The PPV was defined as the number of TB patients with a positive result divided by the total number of positive results (true positives + false positives). The NPV was defined as number of non-TB patients with a negative result divided by the total number of negative results (true negatives + false negatives). Accuracy rate was defined as the proportion of patients for whom test results were consistent with diagnosis. Concordance between the results of the AmpSure and bacteriological analyses was assessed using the Cohen kappa test, with $\kappa>0.75$ indicating excellent agreement, a $\kappa$-value of $0.4-0.75$ indicating moderate agreement, and $\kappa<0.4$ indicating poor agreement. The level of statistical significance was set at $\mathrm{p}<0.05$.

\section{Results}

We prospectively enrolled 442 patients. Four patients for whom a clear diagnosis could not be determined were excluded from our analysis. The demographic characteristics of patients and study group assignment are shown in Table 1. The remaining 438 patients included 269 men and 169 women with a mean age of $47.5 \pm 18.4$ years, among whom 354 patients ( 219 men and 135 women) were confirmed TB cases and 84 patients (50 men and 34 women) had pleural effusion due to an etiology other than TB. There was no statistically significant difference in sex between the TB and non-TB groups $\left(\chi^{2}=0.157, p=0.692\right)$. The TB group was significantly younger ( $43.4 \pm 18.7$ years; range: $17-82$ years) than the non-TB group $(57.2 \pm 14.6$ years; range: $23-82$ years; $\mathrm{p}<0.001)$. Diagnoses for patients in the non-TB group included lung cancer $(\mathrm{n}=10)$, invasive pulmonary fungal infection $(n=1)$, bacterial pneumonia $(n=65)$, sarcoidosis $(n=1)$, pulmonary embolism $(n=2)$, systemic lupus erythematosus $(\mathrm{n}=1)$, and non-tubercle Mycobacterium infection $(\mathrm{n}=4)$.

The results of the various diagnostics tests are summarized in Table 2.

In the TB group, 278 cases were also complicated by pulmonary TB based on sputum analysis. In the analysis of PF, the diagnostic rates for the AFB smear (3.1\%), AmpSure assay (21.2\%), and culture analyses (17.8\%) were lower than that of ADA analysis (100\%) and IFN $\gamma$ release (100\%). The diagnostic rates for the AFB smear, AmpSure assay, and culture analyses of PF were also lower than those of the AFB smear (31.3\%) and culture analyses $(88.1 \%)$ of sputum as well as the histopathological analysis of pleural biopsy (100\%). 


\begin{tabular}{|l|l|l|}
\hline Variable & $\begin{array}{l}\text { TB Group } \\
(\mathbf{N}=354)\end{array}$ & $\begin{array}{l}\text { Non-TB Group } \\
(\mathbf{N}=84)\end{array}$ \\
\hline Men (n [\%]) & $219(61.9)$ & $50(59.5)$ \\
\hline Women (n [\%]) & $135(38.1)$ & $34(40.5)$ \\
\hline Age (y) & $43.4 \pm 18.7$ & $57.2 \pm 14.6$ \\
\hline $\begin{array}{l}\text { Mt-positive sputum or } \\
\text { pleural fluid culture (n [\%]) }\end{array}$ & $288(81.4)$ & $0(0)$ \\
\hline $\begin{array}{l}\text { Caseating granuloma in } \\
\text { pleural biopsy (n [\%]) }\end{array}$ & $66(18.6)$ & $0(0)$ \\
\hline
\end{tabular}

Table 1. Patient characteristics and study group assignment. TB, tuberculosis; Mt, Mycobacterium tuberculosis.

\begin{tabular}{|c|c|c|c|c|c|c|}
\hline \multirow[b]{3}{*}{ Method } & \multicolumn{6}{|c|}{ Diagnostic rate $(\mathbf{n} / \mathrm{N})$} \\
\hline & \multicolumn{2}{|l|}{ Sputum* } & \multicolumn{2}{|l|}{ PF } & \multicolumn{2}{|c|}{ Histopathology } \\
\hline & TB group & Non-TB group & TB group & Non-TB group & TB group & Non TB group \\
\hline AmpSure positive & $208 / 278$ & $0 / 76$ & $75 / 354$ & $0 / 84$ & & \\
\hline AFB smear positive & $87 / 278$ & $2 / 76$ & $11 / 354$ & $0 / 84$ & & \\
\hline Mt-culture positive & $245 / 278$ & $0 / 76$ & $63 / 354$ & $0 / 84$ & & \\
\hline Histopathology-confirmed & & & & & $66 / 66$ & 12 negative/ 12 \\
\hline IFN $\gamma$ release of $\mathrm{PF}(>50 \%)$ & & & $354 / 354$ & $66 / 84$ & & \\
\hline $\mathrm{ADA}$ of $\mathrm{PF}(>40 \mathrm{IU} / \mathrm{L})$ & & & $354 / 354$ & $20 / 84$ & & \\
\hline $\begin{array}{l}\text { AmpSure positive/AFB and } \\
\text { Mt-culture negative }\end{array}$ & & & $35 / 354$ & $0 / 84$ & & \\
\hline $\begin{array}{l}\text { AFB positive/AmpSure and Mt- } \\
\text { culture negative }\end{array}$ & & & $0 / 354$ & $2 / 84$ & & \\
\hline $\begin{array}{l}\text { Mt-culture positive/AmpSure } \\
\text { and AFB negative }\end{array}$ & & & $22 / 354$ & $0 / 84$ & & \\
\hline
\end{tabular}

Table 2. Summary of results of the diagnostic tests. *Sputum from $278 / 354$ TB cases and from $76 / 84$ non-TB cases was examined. AFB, acid-fast bacilli; PF, pleural fluid; Mt, Mycobacterium tuberculosis; IFN $\gamma$, interferon gamma; $\mathrm{ADA}$, adenine deaminase.

\begin{tabular}{|l|l|l|l|l|l|}
\hline Method & Sensitivity & Specificiy & PPV & NPV & Accuracy \\
\hline AmpSure & $21.2 \%$ & $100 \%$ & $100 \%$ & $3.1 \%$ & $36.3 \%$ \\
\hline AFB smear & $3.1 \%^{\mathrm{a}}$ & $96.4 \%$ & $78.6 \%$ & $19.8 \%$ & $21.0 \%$ \\
\hline Mt culture & $17.8 \%^{\mathrm{b}}$ & $100 \%$ & $100 \%$ & $22.4 \%$ & $33.6 \%$ \\
\hline
\end{tabular}

Table 3. Comparison of AmpSure, AFB smear, and culture results for pleural fluid samples. AFB, acid-fast bacilli; PF, pleural fluid; Mt, Mycobacterium tuberculosis; ${ }^{\mathrm{p}} \mathrm{p}<0.001$ compared with AmpSure, ${ }^{\mathrm{b}} \mathrm{p}>0.05$ compared with AmpSure.

The comparison of the diagnostic characteristics of the various methods of $M$. tuberculosis detection is shown in Table 3. The sensitivity of the AmpSure assay for the analysis of PF samples (21.2\%) was significantly higher than that of the AFB smear analysis $\left(3.1 \% ; \chi^{2}=54.2, p<0.001\right)$, but it was not significantly different than the sensitivity of the BACTEC MGIT 960 testing of PF samples $\left(17.8 \% ; \chi^{2}=1.291, \mathrm{p}=0.255\right)$. Both the AmpSure assay and the BACTEC MGIT 960 system demonstrated higher specificities and PPVs for PF than those of the AFB smear analysis, and the NPVs of all of the tests were $<24 \%$. Concordance between the results of the AmpSure assay and the BACTEC MGIT 960 test for sputum for the entire cohort $(\mathrm{N}=438)$ and that of the AmpSure assay and culture method for PF was moderate $(\kappa=0.571, \mathrm{p}<0.001)$. The results suggested that the diagnostic characteristics are suboptimal for the detection of $M$. tuberculosis in PF, and that the characteristics of the BACTEC and smear analyses likely differed between the sputum and PF samples.

\section{Discussion}

In this study, we evaluated three laboratory methods for the diagnosis of TPE in Chinese patients, and found that all were suboptimal for the detection of M. tuberculosis in PF. Similar previous investigations of methods for detecting M. tuberculosis in the PF have also reported suboptimal sensitivities for identifying patients with $\mathrm{TPE}^{3,4,21,22}$. However, the AmpSure diagnostic test has demonstrated high levels of sensitivity and specificity for the detection of M. tuberculosis in sputum samples ${ }^{16}$. Therefore, in our comparison of the diagnostic utility of the AmpSure test for detecting M. tuberculosis in PF to that of the BACTEC MGIT 960 and AFB smear analyses, we found that the sensitivities of these methods are suboptimal for the detection of tubercle bacilli in PF samples, whereas each of them demonstrates high specificity. 
The higher sensitivity of the AmpSure assay for detecting M. tuberculosis in sputum samples, relative to that of AFB smear analysis, suggests that it might also perform better for detecting tubercle bacilli in PF. However, studies have shown that the sensitivities of various methods of direct detection of tubercle bacilli can vary between respiratory and nonrespiratory samples, despite the levels of potential inhibitors of nucleic acid detection, such as RNAases, being present at similar levels ${ }^{23,24}$. Moreover, the results of our direct comparison of AmpSure detection of the $16 \mathrm{~S}$ ribosomal RNA of $M$. tuberculosis, the selective propagation of $M$. tuberculosis in liquid culture medium by the BACTEC MGIT method, and the AFB smear analysis suggest that the factors which contribute to the reduced sensitivities of these methods for PF testing are nonspecific in nature.

In addition, previous studies of other molecular methods of $M$. tuberculosis detection in PF samples have reported higher sensitivities than that which we determined for the AmpSure test in our current study, with sensitivities of $25-43.6 \%$ and $93 \%$ for the Xpert MTB/RIF test ${ }^{25,26}$ (Cepheid, Sunnyvale, CA, USA) and a specific antibody-based detection method ${ }^{27}$, respectively. In addition, one previous study of a PCR-based analysis reported a sensitivity of $93.1 \%{ }^{28}$. However, the specific antibody-based test has not been replicated, and the PCR-based analysis and Xpert MTB/RIF evaluations included a total of only 177 PF samples, whereas our analysis included samples from 438 suspected TP cases. One possible shortcoming of our analysis was the composite index used to confirm TB diagnosis. Clinical examinations and the interpretation of chest radiographs and CT scans are subject to variation due to the expertise and experience of clinicians. However, no "gold standard" exists for the direct detection of tubercle bacilli in PF against which our results could be compared, and the TB diagnostic index we used represented an approach applicable to resource-limited settings in China.

Smear microscopy for the detection of AFB remains the most widely used diagnostic test for M. tuberculosis detection in resource-limited settings around the world. The cost of the Xpert MTB/RIF are prohibitive for most patients in China. The cost of the AmpSure test is, however, a reimbursable expense under China's national insurance program ${ }^{16}$. A study in China found that including the BACTEC MGIT test in routine diagnostic testing for TB patients could be a cost-effective long-term measure ${ }^{29}$. A cost comparison showed that prices for AmpSure, BACTEC and smear test were 14 USD, 22 USD and 2 USD, respectively. Given the high specificities observed for the AmpSure test (100\%), BACTEC MGIT test (100\%), and AFB smear analysis (96.4\%) in our current study, a composite index consisting of these tests might be the most effective strategy for the diagnosis of TP at centralized diagnostic facilities with real-time fluorescence detection instruments in China. The diagnostic rate of the combination of the above three different methods reached $79.1 \%$. Such a composite index would represent a balance between the cost-effectiveness and diagnostic accuracy of these methods in an area where TB burden is high.

\section{References}

1. Global Tuberculosis Report 2016. Vol. 2016 (World Health Organization, 2016).

2. Mehta, J., Dutt, A., Harvill, L. \& Mathews, K. Epidemiology of extrapulmonary tuberculosis. A comparative analysis with pre-AIDS era. Chest 99, 1134 (1991).

3. Light, R. Update on tuberculous pleural effusion. Respirology (Carlton, Vic.) 15, 451 (2010).

4. Valdés, L. et al. Tuberculous pleurisy: a study of 254 patients. Archives of internal medicine 158, 2017 (1998).

5. Norbis, L., Miotto, P., Alagna, R. \& Cirillo, D. Tuberculosis: lights and shadows in the current diagnostic landscape. The new microbiologica 36, $111(2013)$

6. Luzze, H. et al. Evaluation of suspected tuberculous pleurisy: clinical and diagnostic findings in HIV-1-positive and HIV-negative adults in Uganda. The international journal of tuberculosis and lung disease: the official journal of the International Union against Tuberculosis and Lung Disease 5, 746 (2001).

7. Scharer, L. \& McClement, J. Isolation of tubercle bacilli from needle biopsy specimens of parietal pleura. The American review of respiratory disease $\mathbf{9 7 ,} 466(1968)$.

8. Gopi, A., Madhavan, S., Sharma, S. \& Sahn, S. Diagnosis and treatment of tuberculous pleural effusion in 2006. Chest 131, 880 (2007).

9. Yang, L. et al. Analysis of Cytokine Levers in Pleural Effusions of Tuberculous Pleurisy and Tuberculous Empyema. Mediators of Inflammation 2016 (2016).

10. Koşar, F. et al. The comparative value of pleural fluid adenosine deaminase and neopterin levels in diagnostic utility of pleural tuberculosis. Tuberkuloz ve toraks 63, 243 (2015).

11. Valdés, L. et al. Value of adenosine deaminase in the diagnosis of tuberculous pleural effusions in young patients in a region of high prevalence of tuberculosis. Thorax 50, 600-603 (1995).

12. Kim, H. et al. The prevalence of pulmonary parenchymal tuberculosis in patients with tuberculous pleuritis. Chest 129, 1253 (2006).

13. Control, Cf. D., Prevention. Update: Nucleic acid amplification tests for tuberculosis. MMWR. Morbidity and mortality weekly report 49, 593 (2000).

14. Ling, D. I., Flores, L. L., Riley, L. W. \& Pai, M. Commercial Nucleic-Acid Amplification Tests for Diagnosis of Pulmonary Tuberculosis in Respiratory Specimens: Meta-Analysis and Meta-Regression. PLoS ONE 3 (2008).

15. Cui, Z. et al. Novel Real-Time Simultaneous Amplification and Testing Method To Accurately and Rapidly Detect Mycobacterium tuberculosis Complex. Journal of Clinical Microbiology 50, 646 (2012).

16. Yan, L. et al. A Large Cohort Study on the Clinical Value of Simultaneous Amplification and Testing for the Diagnosis of Pulmonary Tuberculosis. Medicine 95, e2597 (2016).

17. Fan, L. et al. Clinical diagnostic performance of the simultaneous amplification and testing methods for detection of the Mycobacterium tuberculosis complex for smear-negative or sputum-scarce pulmonary tuberculosis in China. Chinese medical journal 127, 1863 (2014).

18. Light, R., Macgregor, M., Luchsinger, P. \& Ball, W. Jr Pleural effusions: the diagnostic separation of transudates and exudates. Annals of internal medicine 77, 507 (1972).

19. Guidelines for surveillance of drug resistance in tuberculosis. 4th edn, (World Health Organization, 2009).

20. Meier, T., Eulenbruch, H., Wrighton-Smith, P., Enders, G. \& Regnath, T. Sensitivity of a new commercial enzyme-linked immunospot assay (T SPOT-TB) for diagnosis of tuberculosis in clinical practice. European journal of clinical microbiology \& infectious diseases: official publication of the European Society of Clinical Microbiology 24, 529 (2005).

21. Liu, Y. et al. A combination of the QuantiFERON-TB Gold In-Tube assay and the detection of adenosine deaminase improves the diagnosis of tuberculous pleural effusion. Emerging Microbes \& Infections 5, e83 (2016).

22. Li, L. et al. Identification of Mycobacterium tuberculosis-Specific Th1, Th17 and Th22 Cells Using the Expression of CD40L in Tuberculous Pleurisy. PLoS ONE 6 (2011). 
23. O'Sullivan, C. E., Miller, D. R., Schneider, P. S. \& Roberts, G. D. Evaluation of Gen-Probe Amplified Mycobacterium Tuberculosis Direct Test by Using Respiratory and Nonrespiratory Specimens in a Tertiary Care Center Laboratory. Journal of Clinical Microbiology 40,1723 (2002).

24. Thwaites, G. Advances in the diagnosis and treatment of tuberculous meningitis. Current opinion in neurology 26, 295 (2013).

25. Du, J. et al. Rapid diagnosis of pleural tuberculosis by Xpert MTB/RIF assay using pleural biopsy and pleural fluid specimens. Journal of Research in Medical Sciences: The Official Journal of Isfahan University of Medical Sciences 20, 26 (2015).

26. Friedrich, S. O., von Groote-Bidlingmaier, F. \& Diacon, A. H. Xpert MTB/RIF Assay for Diagnosis of Pleural Tuberculosis. Journal of Clinical Microbiology 49, 4341 (2011).

27. Yari, S. et al. Modified TB rapid test by proteinase K for rapid diagnosis of pleural tuberculosis. APMIS: acta pathologica, microbiologica, et immunologica Scandinavica 124, 201 (2015).

28. Tang, T., Ahmed, S., Musa, M. \& Zainuddin, Z. Rapid detection of Mycobacterium tuberculosis in clinical samples by multiplex polymerase chain reaction (mPCR). World journal of microbiology \& biotechnology 29, 2389 (2013).

29. Du, Y.-C., Chen, Q.-Y. \& Lin, S.-F. Analysis of cost-effectiveness and cost-benefit of tuberculosis control in Fujian province from 2008 to 2010 [J]. China Tropical Medicine 11, 019 (2012).

\section{Acknowledgements}

This study was sponsored by the clinical research of RNA amplification technology in tuberculosis diagnosis and treatment (W2013RNA01).

\section{Author Contributions}

Qing Zhang and Caicun Zhou were responsible for the conception and design of the study. Qing Zhang and Caicun Zhou were responsible for acquisition of data. Qing Zhang and Caicun Zhou performed the data analysis. Qing Zhang and Caicun Zhou drafted the manuscript. All authors participated in interpretation of the findings and all authors read and approved the final version of the manuscript.

\section{Additional Information}

Competing Interests: The authors declare that they have no competing interests.

Publisher's note: Springer Nature remains neutral with regard to jurisdictional claims in published maps and institutional affiliations.

(c) Open Access This article is licensed under a Creative Commons Attribution 4.0 International License, which permits use, sharing, adaptation, distribution and reproduction in any medium or format, as long as you give appropriate credit to the original author(s) and the source, provide a link to the Creative Commons license, and indicate if changes were made. The images or other third party material in this article are included in the article's Creative Commons license, unless indicated otherwise in a credit line to the material. If material is not included in the article's Creative Commons license and your intended use is not permitted by statutory regulation or exceeds the permitted use, you will need to obtain permission directly from the copyright holder. To view a copy of this license, visit http://creativecommons.org/licenses/by/4.0/.

(C) The Author(s) 2017 